\title{
Relato de experiência: aprendizado remoto em meio à pandemia
} Experience report: remote learning through the pandemic

\author{
Manuela Correa Oliveira \\ Universidade do Estado do Rio de Janeiro - UERJ - Rio de Janeiro - Brasil \\ Patrícia Cabral Araújo \\ Universidade do Estado do Rio de Janeiro - UERJ - Rio de Janeiro - Brasil
}

\author{
Roza Luiza Caiado de Castro Accioly
}

Universidade do Estado do Rio de Janeiro - UERJ - Rio de Janeiro - Brasil

Resumo: O presente trabalho é um relato de experiência desenvolvido a partir do trabalho remoto feito com estudantes de ensino básico de uma escola pública do Rio de Janeiro. A ocorrência de uma pandemia mundial não permitiu que as aulas presenciais pudessem ocorrer de forma segura, o que resultou na virtualização do ensino, forçando professore/as e estudantes a se submeterem a atividades síncronas e/ ou assíncronas para dar continuidade ao processo educacional. O objetivo deste trabalho é apresentar de que forma se deu o trabalho de mediação feito por um grupo de professores de inglês, apontando tanto os pontos positivos dessa interação quanto as dificuldades enfrentadas pela equipe. A participação dos estudantes foi essencial para que o projeto de atividades assíncronas fosse constantemente aprimorado. A experiência com o monitoramento da plataforma utilizada pela escola permitiu que a equipe de professore/as adotasse diferentes métodos conforme o projeto se desenvolveu.

Palavras-chave: Ensino. Virtualização. Aprendizado remoto.

Abstract: This paper is an experience report based on remote activities performed by elementary and high school students from a public school in Rio de Janeiro. The occurrence of a worldwide pandemic made impossible for students to safely have faceto-face classes, which resulted in the virtualization of teaching, and forced teachers and students to submit to synchronous and / or asynchronous activities in order to carry on with the educational process. The aim of this report is to present the positive aspects of this interaction, but also the struggles faced by the team. The improvement of the asynchronous activities was possible due to student's participation. Monitoring the platform used by the school allowed teachers to adopt different approaches in the course of the project.

Keywords: Teaching. Virtualization. Remote learning. 


\section{INTRODUÇÃO}

Medidas de distanciamento social tiveram de ser tomadas com a inesperada chegada de uma pandemia causada pelo vírus SARS-CoV-2. No Brasil, um dos países com maiores incidências de contágios e de mortalidade do mundo todo, tais medidas se tornaram urgentes e visavam evitar, na medida do possível, o aumento dessas taxas.

Em meio a crises político-econômicas e medidas governamentais questionáveis diante da pandemia, as semanas que precediam 0 distanciamento social eram de incertezas em diversos setores. No que diz respeito ao ensino, não seria diferente. Tais medidas repercutiram na educação, tanto no ensino básico quanto no ensino superior, de formas variadas. A suspensão completa das aulas presenciais foi a escolha de muitas instituições que priorizaram a saúde de estudantes e funcionários. A partir desta decisão, algumas escolas passaram a adotar alternativas, como o uso de plataformas digitais que possibilitassem atividades síncronas, a fim de que fosse mantida a interação entre professores e estudantes. Todavia, é necessário ressaltar que uma transposição didática do meio presencial para o meio virtual não vem sem questionamentos, principalmente por parte de educadores. Adotar atividades síncronas é partir do princípio de que todos os estudantes possuem equipamento e tecnologia necessários para este fim. Na intenção de manter o compromisso com o ensino, professore/as, relutantes ao uso da tecnologia digital ou não, passaram a fazer uso de ferramentas digitais em prol da educação. Perante as incertezas de um retorno presencial seguro, a equipe de língua inglesa do Instituto de Aplicação Fernando Rodrigues da Silveira (CAp-UERJ) optou por iniciar, em meio à pandemia, um projeto de atividades assíncronas.

A decisão de propor atividades remotas foi tomada por docentes de diversas instituições, docentes que estavam preocupados com a estagnação do ensino, mas também com o aspecto psicológico dos estudantes, tendo em vista a prolongada permanência em casa. No CAp, essa decisão foi acompanhada pelo chamado Contato CApiano, proposta pela qual os estudantes optavam, ou não, por fazerem atividades no meio virtual, mas cientes de que notas não seriam aferidas. Cabe dizer, ainda, que não havia uma obrigação por parte dos professores de aderirem a esse sistema.

Diante do contexto em que se encontrava, a equipe de inglês do CAp optou pela utilização da plataforma AVA (Ambiente Virtual de Aprendizagem), disponível para os estudantes da UERJ e do Instituto de Aplicação da Universidade. Nesta plataforma, a alunos e alunas do $6^{\circ}$ ano ao Ensino Médio eram disponibilizadas diversas atividades de leitura, escrita e escuta para continuarem seus estudos de língua inglesa. No que respeita ao desenho e aplicação do projeto, a equipe de inglês se dividiu em dois grupos: a) grupo de formulação; b) grupo de mediação. A mediação contou com a participação de docentes e estudantes por meio de fóruns, que possibilitavam o levantamento dúvidas e as respostas às mesmas, acesso a questionários e formulários, e envio de arquivos com as respostas para as atividades. ]

A criação das atividades foi norteada pela abordagem cujo fundamento principal é a resolução de um problema. Na próxima seção, descrevemos, brevemente, os fundamentos do projeto a partir do qual a equipe se baseou, denominado de Project-Based Learning ou Aprendizagem Baseada em Projetos (doravante PBL), com ênfase na maneira como se desenvolveu o processo de mediação da plataforma AVA por parte de um grupo de docentes.

\section{METODOLOGIA ADOTADA}

As atividades realizadas na plataforma foram pensadas com foco no PBL. O referencial teórico utilizado para a criação das atividades se baseou em Moursund (2002): Project-Based Learning Using Information Technology (Aprendizagem Baseada em Projetos Usando Tecnologia da Informação). Nos termos do autor, a perspectiva adotada pode ser definida da seguinte maneira:

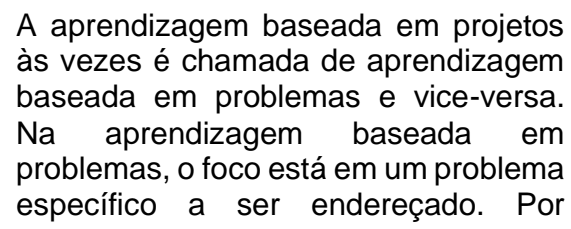


exemplo, o problema pode ser limpar um fluxo poluído percorrendo a cidade ou para salvar espécies ameaçadas de plantas ou animais. ${ }^{1}$ (MOURSUND, 1999, p. 11)

O PBL vem sendo adotado não só no ensino de línguas estrangeiras, mas também por outras disciplinas escolares, visto que contribui para uma aprendizagem significativa e relevante para os estudantes. Um dos fundamentos do PBL se baseia na filosofia da educação construtivista, presumindo-se que o/a estudante é um ser apto para construir novos conhecimentos e relacioná-los com outras bases de conhecimentos prévios que possui. Desta forma, estudantes tem a habilidade de trazerem conhecimentos anteriormente adquiridos para um novo contexto de aprendizagem. Para Sanchis \& Mahfoud (2007), a aprendizagem sob uma educação construtivista significa que:

(...) por um lado, que as estruturas cognitivas do sujeito não estão prontas ao nascer, e por outro, que o sujeito conhece e interpreta o mundo a partir de estruturas próprias, apesar de não serem estanques. A palavra construtivismo se refere exatamente a essa relação entre a estrutura e o processo que permite a transformação da própria estrutura. E esse processo se funda na interação entre o sujeito e o objeto, o que faz com que as estruturas sejam construídas ao mesmo tempo pelos dois, ou melhor, pela relação estabelecida entre eles. A interação é mediada pela ação do sujeito. Ou seja, todo conhecimento está, em todos os níveis, ligado à ação. (SANCHIS \& MAHFOUD, 2007, p. 167).

O PBL se concentra na tarefa de solucionar problemas e para que este resultado seja obtido, é necessária a construção de um trabalho que engloba diferentes tipos de conhecimento provindos de diferentes fontes. Desta maneira, quando diante de um problema ou tarefa desafiadora, o indivíduo faz uso de conhecimentos, habilidades e recursos que outras pessoas desenvolveram, bem como de seus próprios conhecimentos, habilidades e conhecimentos. A construção do conhecimento é conjunta e colaborativa.

1 Project-based learning is sometimes called problem-based learning, and vice versa. In problembased learning, the focus is on a specific problem to be addressed. For example, the problem might be to
Dada a situação emergencial em que docentes e discentes se encontravam, o viés construtivista do PBL pareceu a melhor ferramenta de interação entre professore/as e aluno/as. A equipe de língua inglesa do Cap UERJ decidiu, portanto, levar para frente o projeto como fundamento para o desenho das atividades.

\section{DESENVOLVIMENTO DO PROJETO}

A partir do cenário em que nos encontrávamos e da necessidade de aplicação de atividades de natureza emergencial, as equipes da escola se organizaram para o estabelecimento de algumas diretrizes. No que respeita ao ensino de inglês, a equipe decidiu implementar o PBL e se dividiu em dois grupos: enquanto metade era responsável pelo design das atividades, o outro grupo era responsável pela mediação das mesmas ambiente virtual AVA. O grupo de mediação dava conta de corrigir e avaliar as atividades - sem aferir notas -, responder às mensagens dos fóruns; ajudar aluno/as que tivessem com dificuldade ora com a digitalização de atividades, ora com demandas do nível de inglês, dentre outras atribuições da equipe. Vale ressaltar, porém, que o projeto foi ofertado de maneira facultativa, i.e., o estudante que decidia não participar do projeto não teria risco de reprovação, haja vista o acordo estabelecido entre docentes da instituição de não considerar tais atividades como passíveis de avaliação.

A configuração das turmas de inglês de acordo com escolaridade, total de estudantes e participantes do AVA está representada na tabela 1.

Tabela 1 - Relação de turmas e estudantes de língua inglesa da escola CAp UERJ

\begin{tabular}{|l|l|c|}
\hline $\begin{array}{c}\text { Ano de } \\
\text { escolaridade }\end{array}$ & $\begin{array}{c}\text { Total de } \\
\text { estudantes }\end{array}$ & $\begin{array}{c}\text { Participantes } \\
\text { do AVA }\end{array}$ \\
\hline $6^{\circ}$ & 125 & $104=83 \%$ \\
\hline $7^{\circ}$ & 116 & $93=80 \%$ \\
\hline
\end{tabular}

clean up a polluted stream running through one's city, or to save an endangered species of plant or animal. (MOURSUND, 1999, p. 11) 


\begin{tabular}{|l|l|l|}
\hline $8^{\circ}$ & 121 & $102=84 \%$ \\
\hline $9^{\circ}$ & 122 & $94=77 \%$ \\
\hline $1^{\circ}$ EM & 123 & $58=47 \%$ \\
\hline $2^{\circ}$ EM & 108 & $40=37 \%$ \\
\hline $3^{\circ}$ EM & 82 & $19=23 \%$ \\
\hline
\end{tabular}

A tabela mostra que o número de participantes varia dentre os anos de escolaridade. Ressalta dos dados que a participação do Ensino Médio é significativamente menor se comparada ao Ensino Fundamental. Proporcionalmente, quanto maior o nível de escolaridade, menor parece ser a participação no ambiente virtual. Há uma participação destacadamente maior no que concerne aos anos iniciais da tabela, a saber, $6^{\circ}, 7^{\circ}$ e $8^{\circ}$ anos, passando a ter menor frequência a partir do $9^{\circ}$ ano.

Considerando o fato de que o grupo de mediação contava com três docentes, a seguinte organização foi determinada pela equipe: uma docente era responsável pelo $6^{\circ}$ ano e parte do $7^{\circ}$, enquanto outra docente dava conta do $8^{\circ}$ e $9^{\circ}$ anos, e, por fim, mais uma docente tomava conta dos participantes do Ensino Médio e da outra parte do $7^{\circ}$. A divisão tinha como objetivo equilibrar o número de participantes para cada professora.

Os participantes foram expostos a uma variedade de atividades e que normalmente eram constituídas dos seguintes passos:
a) Agenda do dia;
b) Vocabulary Log;
c) Pre-listening/ reading/ viewing;
d) While listening/ reading/ viewing;
e) Language corner;
f) Post- listening/ reading/ viewing
g) Challenge
h) Before you leave/ Converse com a gente

O tópico da atividade é introduzido na agenda do dia (a) e os participantes contavam com uma forma de glossário (b), constituído de entradas lexicais relevantes para a execução da atividade em questão. Vale ressaltar que esta seção era colaborativa, pois aquele que estava desempenhando a atividade poderia contribuir com outras formas lexicais que julgasse relevantes para o exercício. Tendo em mente o processo de scaffolding, tão caro ao ensino de línguas, estudantes passam para a seção que os preparava para a atividade em si (c e d). Em paralelo a este processo, havia uma seção, denominada de language corner, cujo foco era o contraste entre expressões apresentadas na atividade. Assim, por exemplo, em uma agenda de filmes e séries de TV, a equipe de inglês indiciou a diferença entre as palavras legend (lenda) e subtitile (legenda), a fim de apresentar o conceito de cognatos entre as línguas inglesa e portuguesa. Na seção (f), os participantes eram levados a executar uma tarefa após terem trabalhado o tópico. Com o objetivo de contar ainda com maior engajamento dos estudantes, a equipe propôs a seção challenge (g), que possibilitava a continuidade da tarefa assíncrona por meio de uma ferramenta digital disponibilizada na plataforma, e.g. criação de um jogo da forca, em que estudantes poderiam desafiar uns aos outros ao criarem palavras e definições relacionadas ao tópico proposto na agenda. Finalmente, Before you leave/ Converse com a gente (h) tinha como objetivo abrir espaço para a colocação de dúvidas e/ou comentários a respeito das atividades. A equipe de inglês estava aberta ao feedback dos estudantes, positivos ou negativos, a fim de melhor desenvolver as tarefas futuras, bem como dar suporte aos que necessitavam.

\section{RESULTADOS}

Considerando que alguns problemas servissem de obstáculo para a execução das tarefas por parte de alguns, o resultado daqueles que participavam foi majoritariamente positivo, como as mediadoras puderam perceber a partir dos comentários dos participantes dos fóruns. Além de afirmarem gostar das atividades, mostraram engajamento na execução das mesmas, demonstrando interesse em pesquisar as respostas para os problemas apresentados.

Conforme apresentado na seção 2, pudemos perceber que a participação de aluno/as do Ensino Médio foi consideravelmente menor. Esse baixo índice 
de acesso nos leva a questionar algumas causas que levaram à fraca participação deste segmento. A falta de recursos, como computadores e acesso à internet, é um fator de destaque, pois são ferramentas fundamentais para a participação no ambiente online.

A equipe de mediação pode averiguar que, além da pouca frequência de acesso de alguns grupos, como já apresentado na tabela 1, as professoras se encontravam diante de algumas frustrações por parte dos estudantes. Na seção "Before you leave/ Fale com a gente", por exemplo, um aluno descreveu seu desapontamento diante de uma atividade cujo tema era música e na qual uma das tarefas era selecionar sua parte favorita. O aluno em questão demonstrou frustração pelo fato de não ter conseguido traduzir toda a letra e, portanto, não ter conseguido finalizar a atividade. É necessário destacar que este tipo de desapontamento poderia ter sido facilmente lidado pelo docente se esta fosse uma atividade síncrona. A proximidade temporal, ainda que virtual, entre docente e discente, viabilizaria uma melhor condução da atividade, pois o docente teria a oportunidade de explicar que o objetivo não é compreender palavra por palavra, mas sim o sentido da música de uma forma geral. Dada a inviabilidade deste monitoramento, que permite um auxílio no momento da execução da atividade, a equipe de mediação percebeu que este era um ponto, inerente a atividades assíncronas. Contudo, o retorno de alunos e alunas auxiliou a equipe de língua inglesa do CAp no melhor desenvolvimento de tarefas futuras, sempre tendo em mente que a autonomia deste estudante está em jogo, mas que ela deve ser desenvolvida com maior cautela.

O grupo de mediação também recebeu dúvidas quanto ao processo de anexação de arquivos à plataforma AVA. Por ser um ambiente novo para um grande número de estudantes, aquilo que parecia uma tarefa simples para uns, como anexar um arquivo, acabava por ser motivo de frustração para outros, que desejavam participar, mas se viam diante de um obstáculo meramente tecnológico. Ademais, muitos estudantes não têm computador e são obrigados a acessar o AVA somente pelo celular. Portanto, mais uma vez, uma barreira tecnológica impediu a finalização de algumas tarefas por parte de alguns participantes.

Dados os apontamentos e questionamentos aqui abordados, tanto o grupo de mediação quanto o grupo de criação trabalharam em conjunto para um melhor procedimento das tarefas. A partir do retorno, positivo ou negativo, dos que acessavam a plataforma, a equipe pôde aprimorar as atividades oferecidas no AVA. Ainda que guiada pelo PBL e pelas bases do construtivismo, a equipe viu a necessidade de constante readaptação das atividades para que pudéssemos estabelecer maior engajamento de estudantes perante uma situação de ensino ainda sem precedentes. A mediação foi um processo essencial para melhor desenvolvimento de tarefas e obtenção de melhores resultados dos exercícios feitos pelos participantes.

\section{CONSIDERAÇÕES FINAIS}

O processo de desenvolvimento e mediação de tarefas na plataforma AVA envolveu o trabalho de toda uma equipe cuja união foi essencial para 0 desenvolvimento de um projeto de qualidade. Os resultados obtidos da experiência mostraram que há muito a ser trabalhado quando se trata da proposta de atividades remotas. Um dos problemas apresentados neste relato está relacionado à dificuldade de acesso, tanto de computadores quanto de internet, por parte de alguns estudantes. O projeto nos mostrou que é preciso oferecer atividades que possam abranger 0 maior número de discentes possível, oferecendo desafios interessantes e problematizadores, mas também acessíveis. O grande desafio é encontrar maneiras de estimular um maior engajamento, especialmente no que concerne aos estudantes do EM, cuja participação foi destacadamente mais fraca.

O grupo de mediação concluiu que o constante feedback de aluno/as ajudaram a equipe de inglês do CAp a desenvolverem atividades e projetos mais eficientes. Porém, é de suma importância que estejam disponibilizados, para docentes e discentes, os equipamentos necessários, e que sejam fornecidas as verdadeiras condições de acesso às plataformas 
online de ensino. O distanciamento físico não deve ser impedimento para uma aproximação social no que diz respeito ao ambiente escolar. O contexto social no qual todos foram colocados, ainda sem precedentes, criou barreiras no sistema educacional, mas forçou toda uma comunidade a se adaptar ao "novo normal". Infelizmente, as oportunidades e condições de acesso a importantes requisitos para uma educação de qualidade já não eram normais antes da pandemia, e continuaram "anormais" durante a mesma. As atividades remotas ajudam docentes e discentes a se manterem conectados com a escola, mas ainda é necessário um olhar cauteloso para que as desigualdades de oportunidade no ambiente escolar.

\section{REFERÊNCIAS}

Ambiente Virtual de Aprendizagem do Instituto de Aplicação Fernando Rodrigues da Silveira AVACAP. Disponível em: <http://ava.cap.uerj.br/>. Acesso em: 20 agosto 2020.

MOURSUND, David. Project-Based Learning Using Information Technology. Eugene, University of Oregon: ISTE and Information Age Education, 1999.

SANCHIS, Isabelle de Paiva; MAHFOUD, Miguel. Interação e Construção: o Sujeito e o Conhecimento no Construtivismo de Piaget. Ciências \& Cognição, v. 12, p.165-176, 2007.

WOOD, David. J., BRUNER, Jerome. S., \& ROSS, Gail. The Role of Tutoring in Problem Solving. Journal of Child Psychiatry and Psychology. v. 17, p. 89-100, 1976.

\section{COMO CITAR ESSE ARTIGO}

OLIVEIRA, Manuela Correa; ARAÚJO, Patrícia Cabral; ACCIOLY, Roza Luiza Caiado de Castro. Relato de experiência: aprendizado remoto em meio à pandemia. Signo, Santa Cruz do Sul, v. 46, n. 85, p. 272-277, jan. 2021. ISSN 1982-2014. Disponível em:

<https://online.unisc.br/seer/index.php/signo/article/view/15690>.doi:https://doi.org/10.17058/signo.v46i85.1 5690 . 